\title{
MODELAGEM MATEMÁTICA PARA ANÁLISE DE VIBRAÇÕES EM UM VEÍCULO AUTOMOTOR
}

BEng. Odilon Hítalo Castro Oliveira ${ }^{(1)}$ (hitalo.castro@hotmail.com), BEng. Rodrigo José da Silva ${ }^{(2)}$ (js.rodrigo_@hotmail.com), Dr. Márcio Eduardo Silveira ${ }^{(3)}$ (msilveira@ufsj.edu.br), Dr. Jorge Nei Brito ${ }^{(4)}$ (brito@ufsj.edu.br), Dr. José Angel Dávalos Chuquipoma ${ }^{(5)}$ (jadc13@ufsj.edu.br)

(1) Universidade Federal de São João Del Rei (UFSJ)

(2) Universidade Federal de São João Del Rei (UFSJ); Programa de Pós-Graduação em Engenharia Mecânica - PPMEC

(3) Universidade Federal de São João Del Rei (UFSJ); Departamento de Engenharia Mecânica - DEMEC

(4) Universidade Federal de São João Del Rei (UFSJ); Departamento de Engenharia Mecânica - DEMEC

(5) Universidade Federal de São João Del Rei (UFSJ); Departamento de Matemática - DEMAT

RESUMO: A obtenção de parâmetros ideais para componentes de uma suspensão veicular, através do controle de vibrações, é extremamente necessária para que se possa garantir o conforto dos ocupantes, assim como a integridade dos componentes mecânicos do automóvel. Deste modo, um modelo matemático que represente o sistema sob vibração torna-se fundamental para que uma análise adequada seja efetuada. Dada a situação, este trabalho se propôs à modelagem de um veículo com oito graus de liberdade, através dos princípios básicos da Mecânica Clássica. A partir da idealização do modelo, onde cada um dos componentes foi representado de maneira esquemática, efetuou-se o estabelecimento de hipóteses para a realização dos cálculos. Posteriormente, todos os passos e manipulações matemáticas foram representados e explicados para fins de obtenção das equações de movimento do veículo que, por fim, foram denotadas em forma matricial.

PALAVRAS-CHAVE: VIBRAÇÕES MECÂNICAS, EQUAÇÕES DE MOVIMENTO, VEÍCULO AUTOMOTOR, OITO GRAUS DE LIBERDADE.

\section{MATHEMATICAL MODELING FOR VIBRATIONAL ANALYSIS IN AN AUTOMOTIVE VEHICLE}

ABSTRACT: Obtaining optimal parameters for vehicle's suspension components through vibration control is extremely necessary to ensure occupant comfort as well as the integrity of the car's mechanical components. Thus, a mathematical model that represents the system under vibration becomes fundamental for a proper analysis to be performed. Given the situation, this work proposed the modeling of a vehicle with eight degrees of freedom, through the basic principles of Classical Mechanics. From the idealization of the model, where each of the components was represented in a schematic manner, hypotheses were made for the calculation. Subsequently, all the steps and mathematical manipulations were represented and explained for the purpose of obtaining the vehicle's equations of motion which were finally denoted in matrix form.

KEYWORDS: MECHANICAL VIBRATIONS, EQUATIONS OF MOTION, AUTOMOTIVE VEHICLE, EIGHT DEGREES OF FREEDOM 


\section{$\mathbf{X I X}$ \\ CONEMI}

\section{INTRODUÇÃO}

Vibração é o movimento oscilatório periódico que ocorre em sistemas dinâmicos. Segundo Rao (2008), a maior parte das atividades realizadas pela humanidade envolvem vibrações. E, recentemente, diversas investigações são aguçadas pelas aplicações da vibração na engenharia, como projeto de máquinas, fundações, estruturas, motores, turbinas e sistemas de controle.

O controle de vibrações advindas de um determinado equipamento ou ocorrentes no mesmo devido a forças externas é de vital importância para que este possa ser operado com regularidade, visando tanto o prolongamento da vida útil do aparato quanto o conforto e segurança de quem o opera ou dele faz uso. Uma suspensão automobilística mal projetada ou danificada, pode trazer, aos ocupantes de um veículo, grande desconforto e, inclusive, complicações de saúde, como lombalgia (dor que ocorre na região lombar inferior) e alterações fisiológicas no corpo humano; a vibração não desejada pode provocar, também, mal funcionamento de componentes mecânicos, assim como levalos à falha por fadiga.

\subsection{Modelagem matemática com " $n$ " graus de liberdade}

"A análise da vibração de sistemas contínuos requer a solução de equações diferenciais parciais, o que é bastante difícil. Na verdade, não existem soluções analíticas para muitas equações diferenciais parciais. Por outro lado, a análise de um com muitos graus de liberdade requer a solução de um conjunto de equações diferenciais ordinárias, o que é relativamente simples. Por consequência, para simplicidade da análise, sistemas contínuos são frequentemente aproximados como sistemas com vários graus de liberdade" (Rao, 2007).

O número de equações de movimento será determinado pelo número de graus de liberdade no qual o sistema será modelado. Desta forma, as equações de movimento podem ser representadas por uma equação matricial (Equação 1),

$$
[m]\{\ddot{x}\}+[c]\{\dot{x}\}+[k]\{x\}=\{f\}
$$

onde $[m]$ é a matriz massa, $[c]$ a matriz amortecimento, $[k]$ a matriz rigidez, $\{\ddot{x}\}$ o vetor aceleração, $\{\dot{x}\}$ o vetor velocidade, $\{x\}$ o vetor deslocamento e $\{f\}$ o vetor força externa.

A Figura 1 representa um sistema massa-mola-amortecedor com três graus de liberdade, onde $m$ é a massa, $k$ a rigidez, $c$ o amortecimento, $f(x)$ a força de excitação externa e $x_{1}, x_{2}, x_{3}$ as coordenadas generalizadas. Para fins de análise, supõe-se a hipótese de $x_{3}>x_{2}>x_{1}$. Desta forma, obtêm-se o diagrama de corpo livre, representado na Figura 2: 


\section{XIX \\ CONEMI}

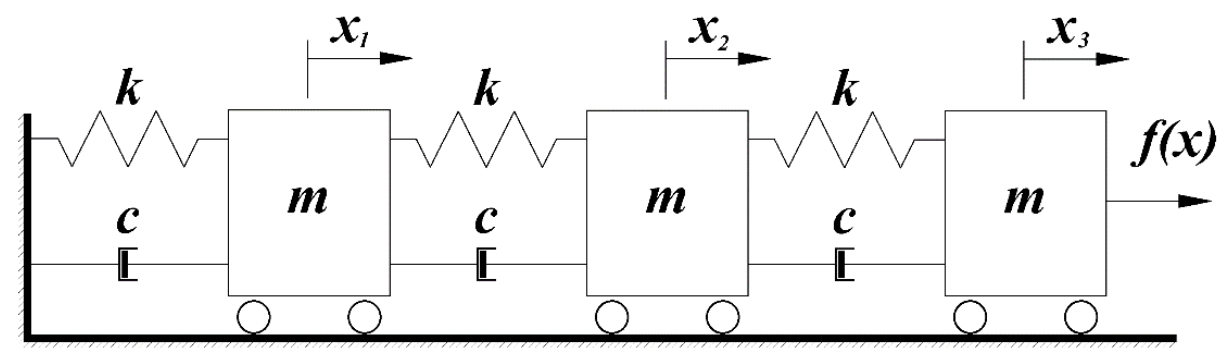

FIGURA 1. Sistema com três graus de liberdade.

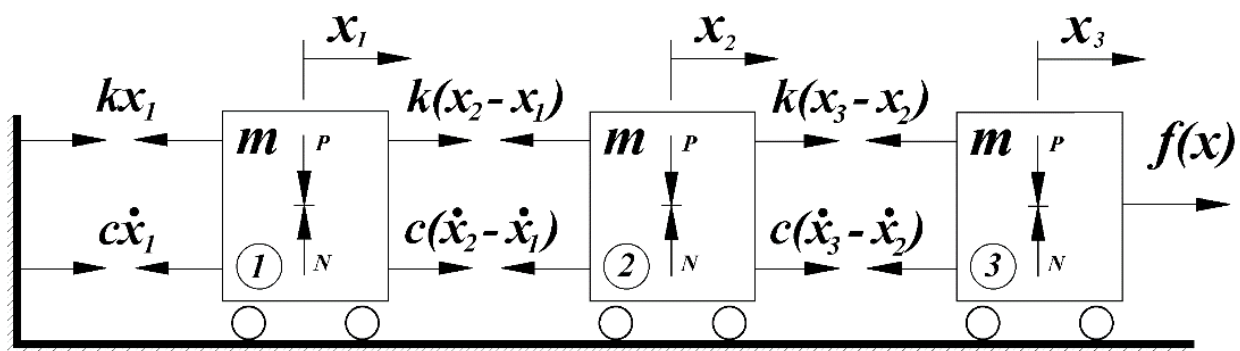

FIGURA 2. Diagrama de corpo livre do sistema.

Pela Segunda Lei de Newton, tem-se (Equação 2):

$$
\overrightarrow{+} \sum F=m \ddot{x}
$$

Sendo assim, obtêm-se equações para os blocos 1 (Equação 3), 2 (Equação 4) e 3 (Equação 5):

$$
\begin{gathered}
-k x_{1}-c \dot{x}_{1}+k\left(x_{2}-x_{1}\right)+c\left(\dot{x}_{2}-\dot{x}_{1}\right)=m \ddot{x}_{1} \\
k\left(x_{2}-x_{1}\right)-c\left(\dot{x}_{2}-\dot{x}_{1}\right)+k\left(x_{3}-x_{2}\right)+c\left(\dot{x}_{3}-\dot{x}_{2}\right)=m \ddot{x}_{2} \\
-k\left(x_{3}-x_{2}\right)-c\left(\dot{x}_{3}-\dot{x}_{2}\right)+f(x)=m \ddot{x}_{3}
\end{gathered}
$$

De forma simplificada, têm-se, pela Equação 6, Equação 7 e Equação 8, as três equações de movimento do sistema, respectivamente:

$$
\begin{gathered}
\left.m \ddot{x}_{1}+2 c \dot{x}_{1}-c \dot{x}_{2}+2 k x_{1}-k x_{2}=0\right\} \\
m \ddot{x}_{2}-c \dot{x}_{1}+2 c \dot{x}_{2}-c \dot{x}_{3}-k x_{1}+2 k x_{2}-k x_{3}=0 \\
m \ddot{x}_{3}-c \dot{x}_{2}+c \dot{x}_{3}-k x_{2}+k x_{3}=f(x)
\end{gathered}
$$

Desta forma, obtêm-se a representação das equações de movimento na forma matricial (Equação 9): 


\section{XIX \\ CENEMI}

$$
\left[\begin{array}{ccc}
m & 0 & 0 \\
0 & m & 0 \\
0 & 0 & m
\end{array}\right]\left\{\begin{array}{l}
\ddot{x}_{1} \\
\ddot{x}_{2} \\
\ddot{x}_{3}
\end{array}\right\}+\left[\begin{array}{ccc}
2 c & -c & 0 \\
-c & 2 c & -c \\
0 & -c & c
\end{array}\right]\left\{\begin{array}{l}
\dot{x}_{1} \\
\dot{x}_{2} \\
\dot{x}_{3}
\end{array}\right\}+\left[\begin{array}{ccc}
2 k & -k & 0 \\
-k & 2 k & -k \\
0 & -k & k
\end{array}\right]\left\{\begin{array}{l}
x_{1} \\
x_{2} \\
x_{3}
\end{array}\right\}=\left\{\begin{array}{c}
0 \\
0 \\
f(x)
\end{array}\right\}
$$

Um sistema real, entretanto, possui infinitos graus de liberdade, fazendo com que a modelagem analítica não seja verdadeiramente precisa, porém aproximada, dependendo do grau de exatidão exigido.

\section{MATERIAIS E MÉTODOS}

A Figura 3 representa a idealização do modelo do veículo com oito graus de liberdade,

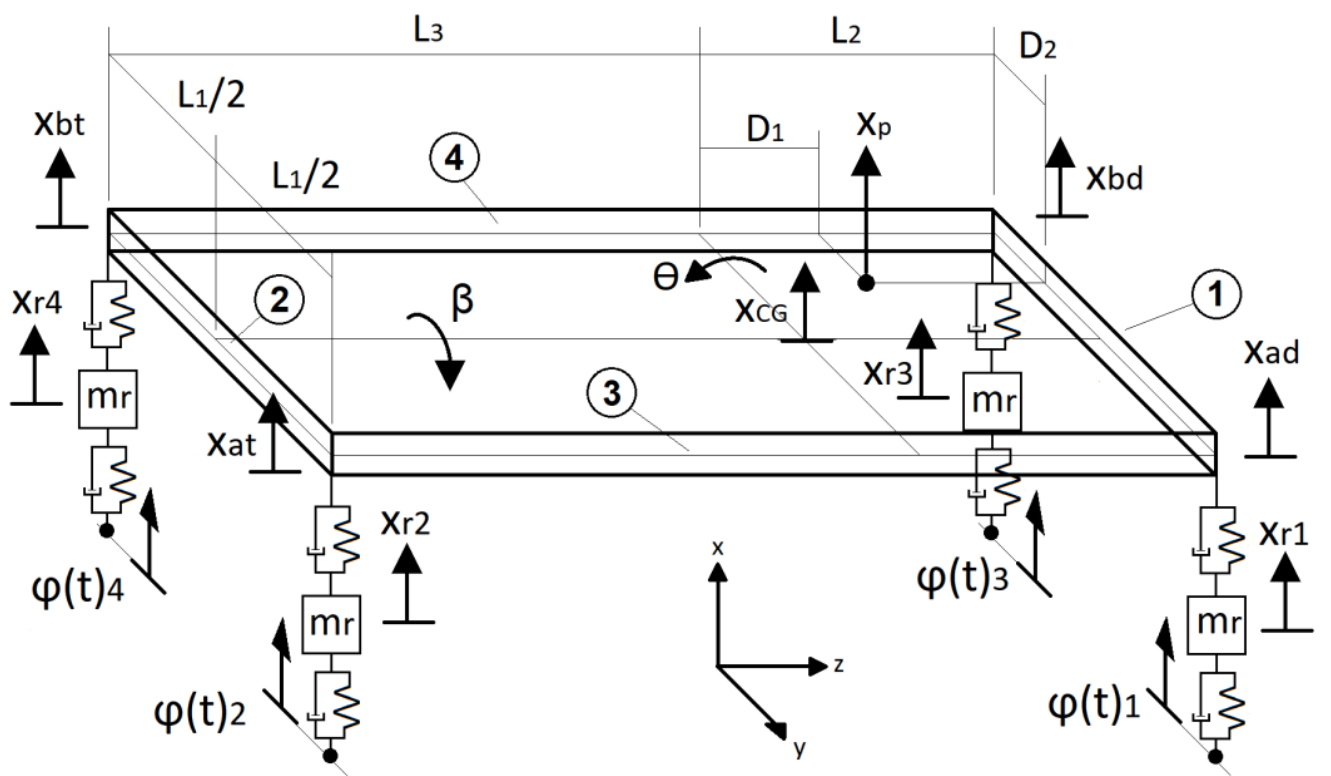

FIGURA 3. Idealização do modelo do veículo com oito graus de liberdade.

onde $\varphi(t)_{1}$ e $\varphi(t)_{2}$ representam as excitações atuantes, respectivamente, sobre os conjuntos pneu/roda dianteiro e traseiro direitos; $\varphi(t)_{3}$ e $\varphi(t)_{4}$ representam as excitações atuantes, respectivamente, sobre os conjuntos pneu/roda dianteiro e traseiro esquerdos; $x_{r 1}$ e $x_{r 2}$ os deslocamentos dos conjuntos pneu/roda, respectivamente, dianteiro e traseiro direitos; $x_{r 3}$ e $x_{r 4}$ os deslocamentos dos conjuntos pneu/roda, respectivamente, dianteiro e traseiro esquerdos; $x_{a d}$ e $x_{a t}$ são as coordenadas de deslocamento das extremidades dianteira e traseiras direitas, respectivamente; $x_{b d}$ e $x_{b t}$ as coordenadas de deslocamento das extremidades dianteira e traseiras esquerdas, respectivamente; $x_{C G}$ a coordenada de deslocamento do centro de gravidade do veículo; $x_{p}$ a coordenada de deslocamento relativa do veículo em relação ao centro de gravidade, do ponto em que se encontra o ocupante; $L_{2}$ e $L_{3}$ representam, respectivamente, as distâncias entre os eixos 


\section{$\mathbf{X I X}$ \\ CENEMI}

dianteiro e esquerdo do veículo a partir do centro de gravidade do mesmo; $L_{1}$ a distância entre os pontos de fixação dos conjuntos mola/amortecedor dos lados direito e esquerdo; $m_{p}$ é massa do ocupante do veículo; $m_{r}$ é a massa de cada conjunto pneu/roda; $D_{1}$ e $D_{2}$ representam as distâncias ao longo do eixo z e eixo $y$, respectivamente, em que o ocupante se encontra do centro de gravidade do veículo; $\theta$ e $\beta$ representam os deslocamentos angulares, em torno do centro de gravidade, de arfagem e deslocamento longitudinal, respectivamente. Os números 1, 2, 3 e 4 indicam, respectivamente, a frente, traseira e as laterais direita e esquerda do veículo.

O modelo leva em consideração: os quatro conjuntos pneu/roda, com as respectivas massas e coeficientes de rigidez e amortecimento dos pneus, fazendo distinção entre pneus traseiros e dianteiros, no que diz respeito às propriedades de rigidez e amortecimento dos mesmos; a suspensão do veículo, representada de maneira independente, considerando os coeficientes de rigidez e amortecimento das molas e amortecedores, fazendo distinções, novamente, entre conjuntos traseiros e dianteiros; o chassi do veículo, representando toda a massa suspensa do mesmo, caracterizado por uma placa de massa $m$ e momentos polares de inércia $J_{1}$, relacionado ao ângulo de rotação $\theta$, e $J_{2}$, relacionado ao ângulo de rotação $\beta$. A Figura 4 representa a idealização do modelo do ocupante do veículo,

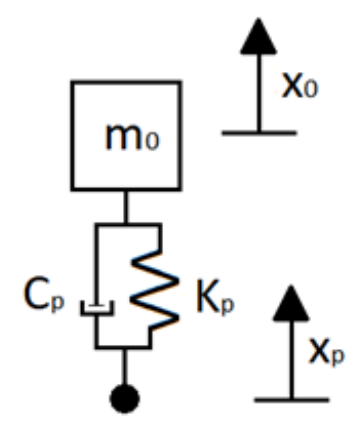

FIGURA 4. Idealização do modelo do ocupante do veículo.

onde $m_{0}$ representa a massa do indivíduo; $C_{p}$ e $K_{p}$ os coeficientes de amortecimento e rigidez do banco do veículo, respectivamente; e $x_{p}$ a coordenada de descolamento do ocupante do veículo.

\subsection{Diagramas de corpo livre e somatórios de forças para os conjuntos pneu/roda}

A Equação 10 representa as hipóteses de desigualdade entre as coordenadas de deslocamento atuantes nos conjuntos pneu/roda dianteiros e traseiros; que, por consequência, aplicam-se às velocidades correlatas em cada coordenada: 


\section{$\mathbf{X I X}$ \\ CENEMI}

$$
\left\{\begin{array} { l } 
{ \varphi ( t ) _ { 1 } > x _ { r 1 } > x _ { a d } } \\
{ \varphi ( t ) _ { 2 } > x _ { r 2 } > x _ { a t } } \\
{ \varphi ( t ) _ { 3 } > x _ { r 3 } > x _ { b d } } \\
{ \varphi ( t ) _ { 4 } > x _ { r 4 } > x _ { b t } }
\end{array} \rightarrow \left\{\begin{array}{l}
\dot{\varphi}(t)_{1}>\dot{x}_{r 1}>\dot{x}_{a d} \\
\dot{\varphi}(t)_{2}>\dot{x}_{r 2}>\dot{x}_{a t} \\
\dot{\varphi}(t)_{3}>\dot{x}_{r 3}>\dot{x}_{b d} \\
\dot{\varphi}(t)_{4}>\dot{x}_{r 4}>\dot{x}_{b t}
\end{array}\right.\right.
$$

A partir da Equação 10, obtém-se os diagramas de corpo livre dos conjuntos pneu/roda do lado direito e esquerdo do veículo, representados, respectivamente, pela Figura 5 e Figura 6,
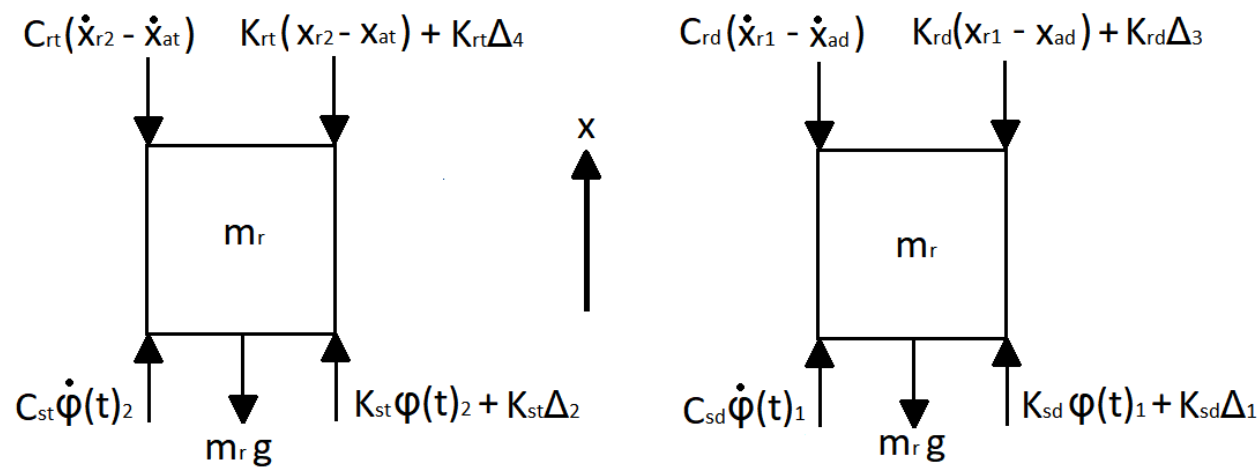

FIGURA 5. Diagramas de corpo livre dos conjuntos pneu/roda direitos.
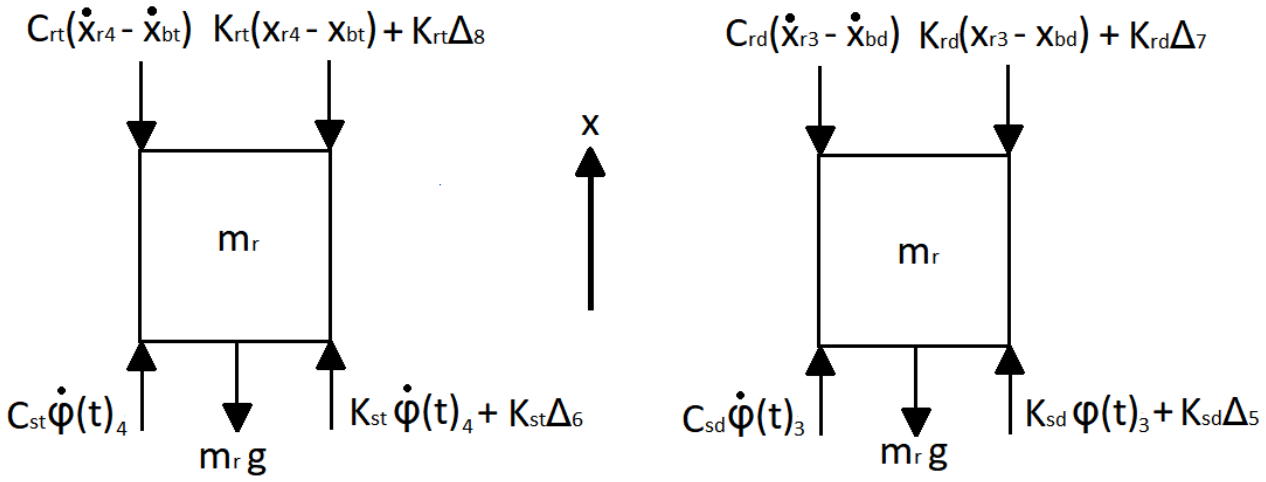

FIGURA 6. Diagramas de corpo livre dos conjuntos pneu/roda esquerdos.

onde $k_{s d}$ e $k_{s t}$ são os coeficientes de rigidez radial do dos pneus dianteiros e traseiros, respectivamente; $c_{s d}$ e $c_{s t}$ são os coeficientes de amortecimento radial do dos pneus dianteiros e traseiros, respectivamente; $k_{r d}$ e $k_{r t}$ os coeficientes de rigidez das molas dianteiras e traseiras, respectivamente, da suspensão do veículo; $c_{r d}$ e $c_{r t}$ os coeficientes de amortecimento dos amortecedores dianteiros e traseiros, respectivamente, da suspensão do veículo; $\Delta_{1}$ e $\Delta_{2}$ são as deformações causadas nos pneus dianteiro e traseiro direitos, respectivamente, pela ação da força normal nas rodas; $\Delta_{3}$ e $\Delta_{4}$ são as deformações causadas nas molas da suspensão do lado direito do veículo, pelos pesos próprio e do ocupante; $\Delta_{5}$ e $\Delta_{6}$ são as deformações causadas nos pneus dianteiro 


\section{$\mathbf{X I X}$ \\ CISNEMI}

e traseiro esquerdos, respectivamente, pela ação da força normal nas rodas; $\Delta_{7}$ e $\Delta_{8}$ são as deformações causadas nas molas da suspensão do lado esquerdo do veículo, devido aos pesos próprio e do ocupante.

Realizando os somatórios de forças nos conjuntos dos lados direito e esquerdo, no equilíbrio estático, tem-se: Equação 11 e Equação 12 para os conjuntos dianteiros e traseiros direitos, respectivamente; Equação 13 e Equação 14 para os conjuntos dianteiros e traseiros esquerdos, respectivamente.

$$
\begin{aligned}
& +\uparrow \sum F=0 \rightarrow k_{s d} \Delta_{1}+k_{r d} \Delta_{3}-m_{r} g=0 \\
& +\uparrow \sum F=0 \rightarrow k_{s t} \Delta_{2}+k_{r t} \Delta_{4}-m_{r} g=0 \\
& +\uparrow \sum F=0 \rightarrow k_{s d} \Delta_{5}+k_{r d} \Delta_{7}-m_{r} g=0 \\
& +\uparrow \sum F=0 \rightarrow k_{s t} \Delta_{6}+k_{r t} \Delta_{8}-m_{r} g=0
\end{aligned}
$$

Para o equilíbrio dinâmico, tem-se: Equação 15 e Equação 16 para os conjuntos dianteiros e traseiros direitos, respectivamente; Equação 17 e Equação 18 para os conjuntos dianteiros e traseiros esquerdos, respectivamente.

$$
\begin{aligned}
& +\uparrow \sum F=m \ddot{x} \rightarrow c_{s d} \dot{\varphi}(t)_{1}+k_{s d} \varphi(t)_{1}-c_{r d}\left(\dot{x}_{r 1}-\dot{x}_{a d}\right)-k_{r d}\left(x_{r 1}-x_{a d}\right) \\
& +k_{s d} \Delta_{1}+k_{r d} \Delta_{3}-m_{r} g=m_{r} \ddot{x}_{r 1} \\
& +\uparrow \sum F=m \ddot{x} \rightarrow c_{s t} \dot{\varphi}(t)_{2}+k_{s t} \varphi(t)_{1}-c_{r t}\left(\dot{x}_{r 2}-\dot{x}_{a t}\right)-k_{r t}\left(x_{r 2}-x_{a t}\right)+k_{s t} \Delta_{2} \\
& +k_{r t} \Delta_{4}-m_{r} g=m_{r} \ddot{x}_{r 2} \\
& +\uparrow \sum F=m \ddot{x} \rightarrow c_{s d} \dot{\varphi}(t)_{3}+k_{s d} \varphi(t)_{3}-c_{r d}\left(\dot{x}_{r 3}-\dot{x}_{b d}\right)-k_{r d}\left(x_{r 3}-x_{b d}\right) \\
& +k_{s d} \Delta_{5}+k_{r d} \Delta_{7}-m_{r} g=m_{r} \ddot{x}_{r 3} \\
& +\uparrow \sum F=m \ddot{x} \rightarrow c_{s t} \dot{\varphi}(t)_{4}+k_{s t} \varphi(t)_{4}-c_{r t}\left(\dot{x}_{r 4}-\dot{x}_{b t}\right)-k_{r t}\left(x_{r 4}-x_{b t}\right)+k_{s t} \Delta_{6} \\
& +k_{r t} \Delta_{8}-m_{r} g=m_{r} \ddot{x}_{r 4}
\end{aligned}
$$

\subsection{Diagramas de corpo livre e somatórios de forças para o ocupante do veículo}

A Equação 19 estabelece as hipóteses de desigualdade entre as coordenadas de deslocamento do ocupante e do chassi do veículo, onde se encontra o mesmo:

$$
\left\{\begin{array}{l}
x_{p}>x_{0} \\
\dot{x}_{p}>\dot{x}_{0}
\end{array}\right.
$$


Deste modo, obtêm-se o diagrama de corpo livre do ocupante do veículo (Figura 7),

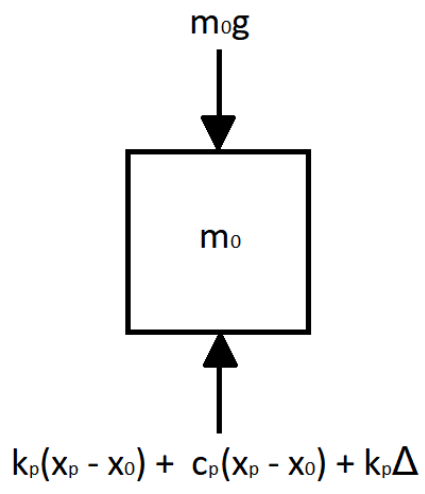

FIGURA 7. Idealização do modelo do ocupante do veículo.

onde $\Delta$ é o deslocamento so banco provocado pelo peso do ocupante.

As equações (20) e (21) representam os somatórios de força no ocupante do veículo no equilíbrio estático e dinâmico, respectivamente:

$$
\begin{gathered}
+\uparrow \sum F=0 \rightarrow k_{p} \Delta-m_{0} g=0 \leftrightarrow k_{p} \Delta=m_{0} g \\
+\uparrow \sum F=m \ddot{x} \rightarrow k_{p} \Delta-m_{0} g+k_{p}\left(x_{p}-x_{0}\right)+c_{p}\left(\dot{x}_{p}-\dot{x}_{0}\right)=m_{0} \ddot{x}_{0}
\end{gathered}
$$

2.3. Diagramas de corpo livre e somatórios de forças para o chassi do veículo e.

A Figura 8 representa o diagrama de corpo livre do chassi do veículo,

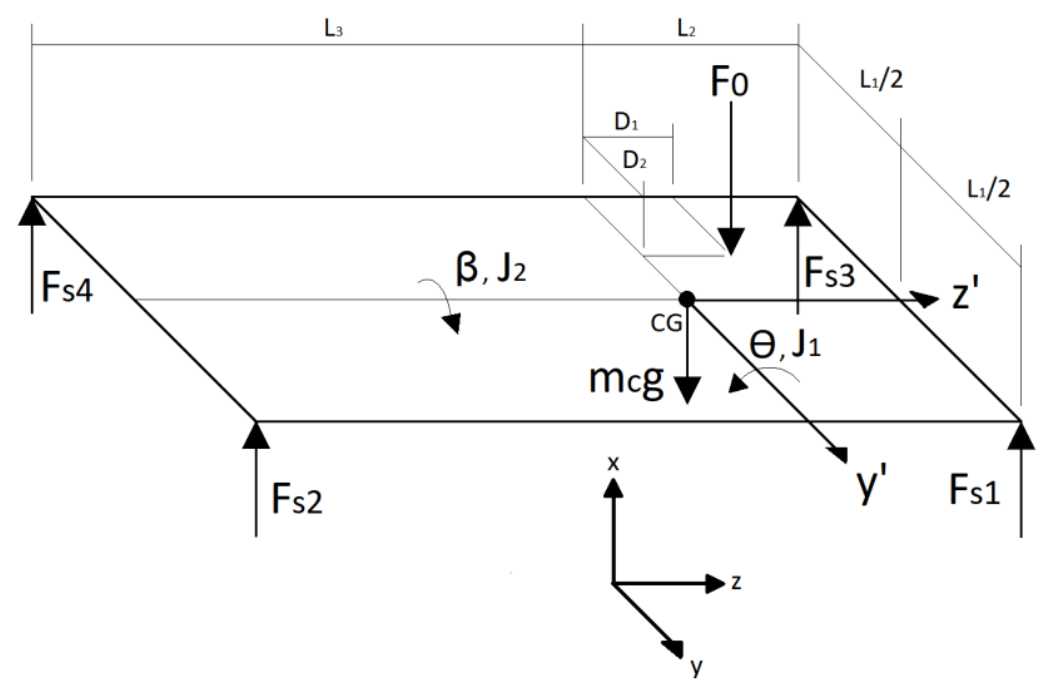

FIGURA 8. Diagrama de corpo livre do chassi do veículo. 


\section{$\mathbf{X I X}$ \\ CIONEMI}

onde $y^{\prime}$ e $z^{\prime}$ são eixos relativos ao centro de gravidade; $J_{1}$ e $J_{2}$ são os momentos polares de inércia do veículo em relação aos eixos y' e $z^{\prime}$, respectivamente; $m_{c}$ é a massa do veículo; $F_{s 1}$ e $F_{s 2}$ são as forças exercidas pelos conjuntos mola/amortecedor dianteiro e traseiro, respectivamente, do lado direito do veículo; $F_{s 3}$ e $F_{s 4}$ são as forças exercidas pelos conjuntos mola/amortecedor dianteiro e traseiro, respectivamente, do lado esquerdo do veículo; $F_{0}$ é a força que o ocupante exerce sobre o chassi veículo; e $g$ é a aceleração da gravidade.

As equações (22), (23), (24), (25) e (26) representam, respectivamente, $F_{s 1}, F_{s 2}, F_{s 3}, F_{s 4}$ e $F_{0}$ :

$$
\begin{gathered}
F_{s 1}=k_{r d}\left(x_{r 1}-x_{a d}\right)+c_{r d}\left(\dot{x}_{r 1}-\dot{x}_{a d}\right)+k_{r d} \Delta_{3} \\
F_{s 2}=k_{r t}\left(x_{r 2}-x_{a t}\right)+c_{r t}\left(\dot{x}_{r 2}-\dot{x}_{a t}\right)+k_{r t} \Delta_{4} \\
F_{s 3}=k_{r d}\left(x_{r 3}-x_{b d}\right)+c_{r d}\left(\dot{x}_{r 3}-\dot{x}_{b d}\right)+k_{r d} \Delta_{7} \\
F_{s 4}=k_{r t}\left(x_{r 4}-x_{b t}\right)+c_{r t}\left(\dot{x}_{r 4}-\dot{x}_{b t}\right)+k_{r t} \Delta_{8} \\
F_{0}=k_{p}\left(x_{p}-x_{0}\right)+c_{r t}\left(\dot{x}_{p}-\dot{x}_{0}\right)+k_{p} \Delta
\end{gathered}
$$

As equações (27), (28), (29) representam os somatórios de força momento no chassi do veículo, respectivamente, no equilíbrio estático, onde é considerada a hipótese de pequenas vibrações, em que $\cos \theta \approx 1$ para as equações de momento;

$$
\begin{gathered}
+\uparrow \sum F=0 \rightarrow k_{r d} \Delta_{3}+k_{r t} \Delta_{4}+k_{r d} \Delta_{7}+k_{r t} \Delta_{8}-k_{p} \Delta-m_{c} g=0 \\
\sum M_{y \prime}=0 \rightarrow k_{r d} \Delta_{3} L_{2}-k_{r t} \Delta_{4} L_{3}+k_{r d} \Delta_{7} L_{2}-k_{r t} \Delta_{8} L_{3}-k_{p} \Delta D_{1}=0 \\
\sum M_{z^{\prime}}=0 \rightarrow\left(-k_{r d} \Delta_{3}-k_{r t} \Delta_{4}+k_{r d} \Delta_{7}+k_{r t} \Delta_{8}\right) \frac{L_{1}}{2}-k_{p} \Delta\left(\frac{L_{1}}{2}-D_{2}\right)=0
\end{gathered}
$$

as equações (30), (31) e (32) no equilíbrio dinâmico:

$$
\begin{aligned}
+\uparrow \sum F=m \ddot{x} & \rightarrow c_{r d}\left(\dot{x}_{r 1}-\dot{x}_{a d}\right)+k_{r d}\left(x_{r 1}-x_{a d}\right)+c_{r t}\left(\dot{x}_{r 2}-\dot{x}_{a t}\right) \\
& +k_{r t}\left(x_{r 2}-x_{a t}\right)+c_{r d}\left(\dot{x}_{r 3}-\dot{x}_{b d}\right)+k_{r d}\left(x_{r 3}-x_{b d}\right) \\
& +c_{r t}\left(\dot{x}_{r 4}-\dot{x}_{b t}\right)+k_{r t}\left(x_{r 4}-x_{b t}\right)+k_{r d} \Delta_{3}+k_{r t} \Delta_{4}+k_{r d} \Delta_{7} \\
& +k_{r t} \Delta_{8}-k_{p} \Delta-m_{c} g=m_{c} \ddot{x}_{C G}
\end{aligned}
$$




\section{XIX \\ CONEMI}

$$
\begin{aligned}
\sum M_{y^{\prime}}=J_{1} \ddot{\theta} & \rightarrow\left[c_{r d}\left(\dot{x}_{r 1}-\dot{x}_{a d}\right)+k_{r d}\left(x_{r 1}-x_{a d}\right)\right] L_{2}-\left[c_{r t}\left(\dot{x}_{r 2}-\dot{x}_{a t}\right)\right. \\
& \left.+k_{r t}\left(x_{r 2}-x_{a t}\right)\right] L_{3}+\left[c_{r d}\left(\dot{x}_{r 3}-\dot{x}_{b d}\right)+k_{r d}\left(x_{r 3}-x_{b d}\right)\right] L_{2} \\
& -\left[c_{r t}\left(\dot{x}_{r 4}-\dot{x}_{b t}\right)+k_{r t}\left(x_{r 4}-x_{b t}\right)\right] L_{3}-\left[c_{p}\left(\dot{x}_{p}-\dot{x}_{0}\right)+k_{p}\left(x_{p}\right.\right. \\
& \left.\left.-x_{0}\right)\right] D_{1}+k_{r d} \Delta_{3} L_{2}-k_{r t} \Delta_{4} L_{3}+k_{r d} \Delta_{7} L_{2}-k_{r t} \Delta_{8} L_{3}-k_{p} \Delta D_{1} \\
& =J_{1} \ddot{\theta}
\end{aligned}
$$

\subsection{Relação entre variáveis}

Para se obter as equações de movimento apenas em função das variáveis desejáveis, faz-se necessário o estabelecimento de relações entre elas, quando estas existem. As equações (33) e (34) representam as relações de igualdade entre as variáveis do modelo:

$$
\begin{gathered}
x_{p}=x_{C G}+\theta D_{1}+\beta\left(\frac{L_{1}}{2}-D_{2}\right) \leftrightarrow x_{C G}=x_{p}-\theta D_{1}-\beta\left(\frac{L_{1}}{2}-D_{2}\right) \\
\left\{\begin{array}{l}
x_{a d}=x_{C G}+\theta L_{2}-\beta \frac{L_{1}}{2} \\
x_{b d}=x_{C G}+\theta L_{2}+\beta \frac{L_{1}}{2} \\
x_{a t}=x_{C G}-\theta L_{3}-\beta \frac{L_{1}}{2} \\
x_{b t}=x_{C G}-\theta L_{3}+\beta \frac{L_{1}}{2}
\end{array}\right.
\end{gathered}
$$

Substituindo a Equação 33 na Equação 34, tem-se a relação desejada entre as os deslocamentos do modelo (Equação 35): 


\section{$\mathbf{X I X}$ \\ CIONEMI}

$$
\left\{\begin{array}{c}
x_{a d}=x_{p}+\left(L_{2}-D_{1}\right) \theta+\left(D_{2}-L_{1}\right) \beta \\
x_{b d}=x_{p}+\left(L_{2}-D_{1}\right) \theta+D_{2} \beta \\
x_{a t}=x_{p}-\left(L_{3}-D_{1}\right) \theta+\left(D_{2}-L_{1}\right) \beta \\
x_{b t}=x_{p}-\left(L_{3}-D_{1}\right) \theta+D_{2} \beta
\end{array}\right.
$$

Derivando a Equação 35 em relação ao tempo, obtêm-se as velocidades (Equação 36):

$$
\left\{\begin{array}{c}
\dot{x}_{a d}=\dot{x}_{p}+\left(L_{2}-D_{1}\right) \dot{\theta}+\left(D_{2}-L_{1}\right) \dot{\beta} \\
\dot{x}_{b d}=\dot{x}_{p}+\left(L_{2}-D_{1}\right) \dot{\theta}+D_{2} \dot{\beta} \\
\dot{x}_{a t}=\dot{x}_{p}-\left(L_{3}-D_{1}\right) \dot{\theta}+\left(D_{2}-L_{1}\right) \dot{\beta} \\
\dot{x}_{b t}=\dot{x}_{p}-\left(L_{3}-D_{1}\right) \dot{\theta}+D_{2} \dot{\beta}
\end{array}\right.
$$

Da mesma forma, derivando a Equação 33 em relação ao tempo, obtêm-se a seguinte relação para a aceleração (Equação 37):

$$
\ddot{x}_{C G}=\ddot{x}_{p}-\ddot{\theta} D_{1}-\ddot{\beta}\left(\frac{L_{1}}{2}-D_{2}\right)
$$

\section{RESULTADOS}

As equações de movimentos são obtidas pela substituição das variáveis de interesse, estabelecidas na Seção 2.4, nas equações de somatório de forças e momentos de cada elemento do modelo do veículo; além da substituição dos somatórios de forças e momentos do equilíbrio estático nas equações do equilíbrio dinâmico.

Substituindo a Equação 20 na Equação 21 e reorganizando-a, obtêm-se a equação de movimento para o ocupante do veículo (Equação 38):

$$
m_{0} \ddot{x}_{0}+c_{p} \dot{x}_{0}-c_{p} \dot{x}_{p}+k_{p} x_{0}-k_{p} x_{p}=0
$$

Para os conjuntos pneu/roda, substitui-se as equações (35), (36) e (37), referentes às variáveis de interesse, nas equações de somatório de forças (15), (16), (17) e (18); para os termos estáticos, substitui-se a Equação 11 na Equação 15, Equação 12 na Equação 16, Equação 13 na Equação 17 e Equação 14 na Equação 18. Após a substituição e devidas reorganizações, obtêm-se a Equação 39 e Equação 40 para os conjuntos pneu/roda dianteiro e traseiro direitos, respectivamente, e a Equação 41 e Equação 42 para os conjuntos pneu/roda dianteiro e traseiro esquerdos, respectivamente: 


\section{$\mathbf{X I X}$ \\ CIONEMI}

$$
\begin{gathered}
m_{r} \ddot{x}_{r 1}+c_{r d} \dot{x}_{r 1}-c_{r d} \dot{x}_{p}-c_{r d}\left(L_{2}-D_{1}\right) \dot{\theta}-c_{r d}\left(D_{2}-L_{1}\right) \dot{\beta}+k_{r d} x_{r 1}-k_{r d} x_{p} \\
-k_{r d}\left(L_{2}-D_{1}\right) \theta-k_{r d}\left(D_{2}-L_{1}\right) \beta=c_{s d} \dot{\varphi}(t)_{1}+k_{s d} \varphi(t)_{1} \\
m_{r} \ddot{x}_{r 2}+c_{r t} \dot{x}_{r 2}-c_{r t} \dot{x}_{p}+c_{r t}\left(L_{3}+D_{1}\right) \dot{\theta}-c_{r t}\left(D_{2}-L_{1}\right) \dot{\beta}+k_{r t} x_{r 2}-k_{r t} x_{p} \\
+k_{r t}\left(L_{3}+D_{1}\right) \theta-k_{r t}\left(D_{2}-L_{1}\right) \beta=c_{s t} \dot{\varphi}(t)_{2}+k_{s t} \varphi(t)_{2} \\
m_{r} \ddot{x}_{r 3}+c_{r d} \dot{x}_{r 3}-c_{r d} \dot{x}_{p}-c_{r d}\left(L_{2}-D_{1}\right) \dot{\theta}-c_{r d} D_{2} \dot{\beta}+k_{r d} x_{r 3}-k_{r d} x_{p} \\
-k_{r d}\left(L_{2}-D_{1}\right) \theta-k_{r d} D_{2} \beta=c_{s d} \dot{\varphi}(t)_{3}+k_{s d} \varphi(t)_{3} \\
m_{r} \ddot{x}_{r 4}+c_{r t} \dot{x}_{r 4}-c_{r t} \dot{x}_{p}+c_{r t}\left(L_{3}+D_{1}\right) \dot{\theta}-c_{r t} D_{2} \dot{\beta}+k_{r t} x_{r 4}-k_{r t} x_{p} \\
+k_{r t}\left(L_{3}+D_{1}\right) \theta-k_{r t} D_{2} \beta=c_{s t} \dot{\varphi}(t)_{4}+k_{s t} \varphi(t)_{4}
\end{gathered}
$$

Para o chassi do veículo, de forma análoga, para as variáveis de interesse, substitui-se as equações (35), (36) e (37) na Equação 30; (35) e (36) nas equações (31) e (32); e, para os termos estáticos, Equação 27 na Equação 30, Equação 28 na Equação 31 e Equação 29 na Equação 32. Reorganizando-as, as equações (43), (44) e (45) representam as equações de movimento do chassi do veículo:

$$
\begin{aligned}
-m_{c} \ddot{x}_{p}+ & m_{c} D_{1} \ddot{\theta}+m_{c}\left(\frac{L_{1}}{2}-D_{2}\right) \ddot{\beta}+c_{r d} \dot{x}_{r 1}+c_{r t} \dot{x}_{r 2}+c_{r d} \dot{x}_{r 3}+c_{r t} \dot{x}_{r 4} \\
& -2\left[\left(c_{r d}+c_{r t}\right)+c_{p}\right] \dot{x}_{p}+c_{p} \dot{x}_{0} \\
& +2\left[c_{r d}\left(D_{1}-L_{2}\right)+c_{r t}\left(L_{3}+D_{1}\right)\right] \dot{\theta} \\
& +\left[\left(L_{1}-2 D_{2}\right)\left(c_{r d}+c_{r t}\right)\right] \dot{\beta}+k_{r d} x_{r 1}+k_{r t} x_{r 2}+k_{r d} x_{r 3} \\
& +k_{r t} x_{r 4}-2\left[\left(k_{r d}+k_{r t}\right)+k_{p}\right] x_{p}+k_{p} x_{0} \\
& +2\left[k_{r d}\left(D_{1}-L_{2}\right)+k_{r t}\left(L_{3}+D_{1}\right)\right] \theta \\
& +\left[\left(L_{1}-2 D_{2}\right)\left(k_{r d}+k_{r t}\right)\right] \beta=0 \\
\frac{2 J_{2}}{L_{1}} \ddot{\beta}+c_{r d} \dot{x}_{r 1} & +c_{r t} \dot{x}_{r 2}-c_{r d} \dot{x}_{r 3}-c_{r t} \dot{x}_{r 4}+L_{1}\left(c_{r d}+c_{r t}\right) \dot{\beta}+k_{r d} x_{r 1}+k_{r t} \dot{x}_{r 2} \\
& -k_{r d} x_{r 3}-k_{r t} x_{r 4}+L_{1}\left(k_{r d}+k_{r t}\right) \beta+c_{p}\left(1-\frac{2 D_{2}}{L_{1}}\right) \dot{x}_{p} \\
& -c_{p}\left(1-\frac{2 D_{2}}{L_{1}}\right) \dot{x}_{0}+k_{p}\left(1-\frac{2 D_{2}}{L_{1}}\right) x_{p}-k_{p}\left(1-\frac{2 D_{2}}{L_{1}}\right) x_{0}=0
\end{aligned}
$$




\section{XIX \\ CENEMI}

$$
\begin{aligned}
J_{1} \ddot{\theta}-c_{r d} L_{2} \dot{x}_{r 1} & +c_{r t} L_{3} \dot{x}_{r 2}-c_{r d} L_{2} \dot{x}_{r 3}+c_{r t} L_{3} \dot{x}_{r 4}+\left[2\left(c_{r d} L_{2}-c_{r t} L_{3}\right)-c_{p} D_{1}\right] \dot{x}_{p} \\
& +2\left[c_{r d} L_{2}\left(L_{2}-D_{1}\right)+c_{r t} L_{3}\left(L_{3}+D_{1}\right)\right] \dot{\theta} \\
& +\left\{c_{r d} L_{2}\left[\left(D_{1}-L_{2}\right)+L_{3} D_{2}\right]-c_{r t} L_{3}\left(2 D_{2}-L_{1}\right)\right\} \dot{\beta}-k_{r d} L_{2} x_{r 1} \\
& +k_{r t} L_{3} \dot{x}_{r 2}-k_{r d} L_{2} x_{r 3}+k_{r t} L_{3} x_{r 4}+\left[2\left(k_{r d} L_{2}-k_{r t} L_{3}\right)-k_{p} D_{1}\right] x_{p} \\
& +2\left[k_{r d} L_{2}\left(L_{2}-D_{1}\right)+k_{r t} L_{3}\left(L_{3}+D_{1}\right)\right] \Theta \\
& +\left\{k_{r d}\left[L_{2}\left(D_{1}-L_{2}\right)+L_{3} D_{2}\right]-k_{r t} L_{3}\left(2 D_{2}-L_{1}\right)\right\} \dot{\beta}+c_{p} D_{1} \dot{x}_{0} \\
& +k_{p} D_{1} x_{0}=0
\end{aligned}
$$

Os termos $a_{i}$ e $b_{i}$, expressos pela Equação 46, representam a redução dos termos das equações de movimento, a fim de se obter um melhor ajuste na expressão matricial:

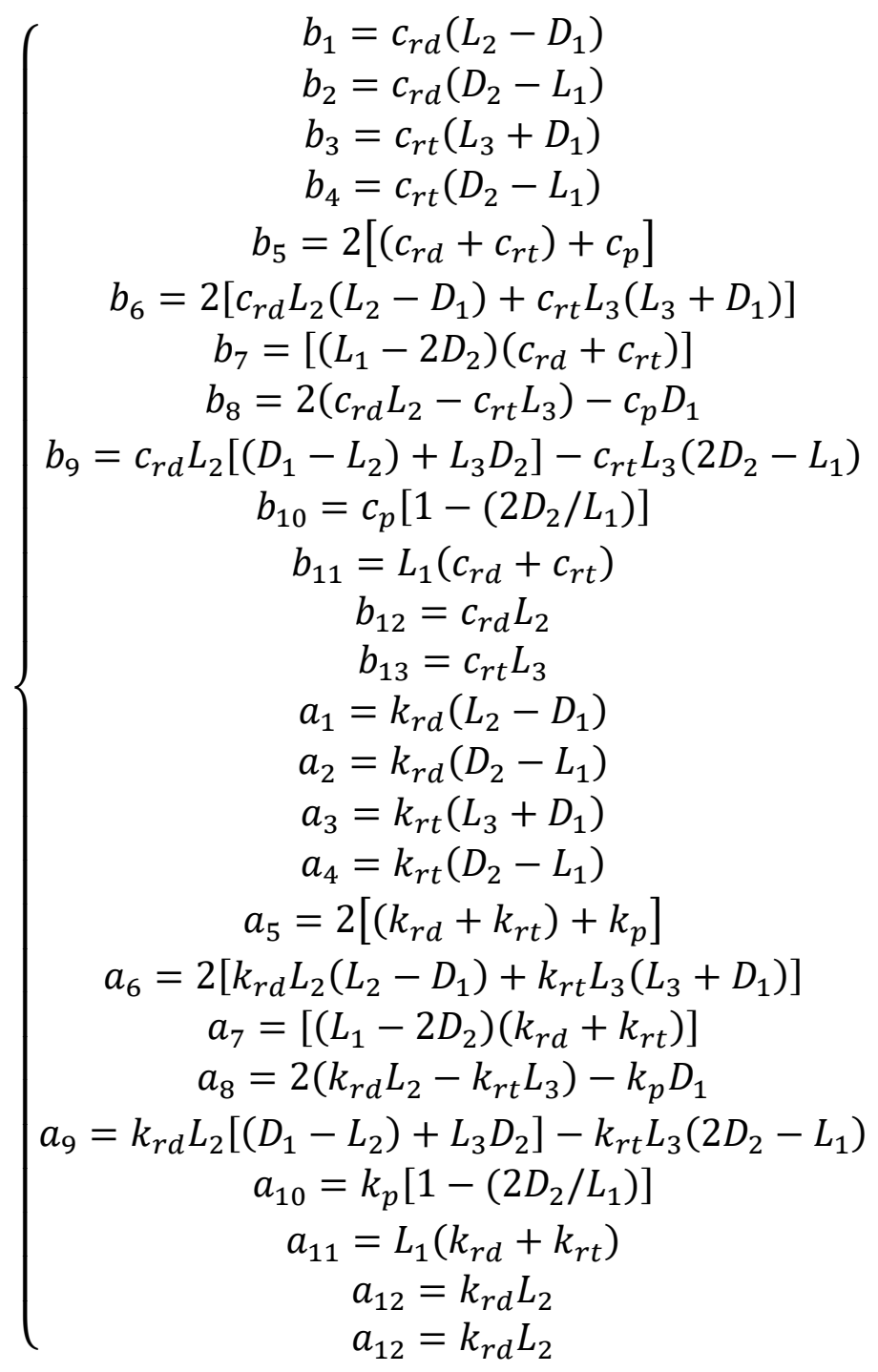


A Equação 47 representa as equações de movimento do veículo na forma matricial:

$\left[\begin{array}{cccccccc}0 & 0 & 0 & 0 & 0 & m_{0} & 0 & 0 \\ m_{r} & 0 & 0 & 0 & 0 & 0 & 0 & 0 \\ 0 & m_{r} & 0 & 0 & 0 & 0 & 0 & 0 \\ 0 & 0 & m_{r} & 0 & 0 & 0 & 0 & 0 \\ 0 & 0 & 0 & m_{r} & 0 & 0 & 0 & 0 \\ 0 & 0 & 0 & 0 & -m_{c} & 0 & m_{c} D_{1} & m_{c}\left(\frac{L_{1}}{2}-D_{2}\right) \\ 0 & 0 & 0 & 0 & 0 & 0 & J_{1} & 0 \\ 0 & 0 & 0 & 0 & 0 & 0 & 0 & \frac{2 J_{2}}{L_{1}}\end{array}\right]\left\{\begin{array}{c}\ddot{x}_{r 1} \\ \ddot{x}_{r 2} \\ \ddot{x}_{r 3} \\ \ddot{x}_{r 4} \\ \ddot{x}_{p} \\ \ddot{x}_{0} \\ \ddot{\theta} \\ \ddot{\beta}\end{array}\right\}$

$+\left[\begin{array}{cccccccc}0 & 0 & 0 & 0 & -c_{p} & -c_{p} & 0 & 0 \\ c_{r d} & 0 & 0 & 0 & -c_{r d} & 0 & -b_{1} & -b_{2} \\ 0 & c_{r t} & 0 & 0 & -c_{r t} & 0 & b_{3} & -b_{4} \\ 0 & 0 & c_{r d} & 0 & -c_{r d} & 0 & b_{1} & -c_{r d} D_{2} \\ 0 & 0 & 0 & c_{r t} & -c_{r t} & 0 & b_{3} & -c_{r t} D_{2} \\ c_{r d} & c_{r t} & c_{r d} & c_{r t} & -b_{5} & c_{p} & b_{6} & b_{7} \\ -b_{12} & b_{13} & -b_{12} & b_{13} & b_{8} & c_{p} D_{1} & b_{6} & b_{9} \\ c_{r d} & c_{r t} & -c_{r d} & -c_{r t} & b_{10} & -b_{10} & 0 & b_{11}\end{array}\right]\left\{\begin{array}{c}\dot{x}_{r 1} \\ \dot{x}_{r 2} \\ \dot{x}_{r 3} \\ \dot{x}_{r 4} \\ \dot{x}_{p} \\ \dot{x}_{0} \\ \dot{\theta} \\ \dot{\beta}\end{array}\right\}$

$+\left[\begin{array}{cccccccc}0 & 0 & 0 & 0 & -k_{p} & -k_{p} & 0 & 0 \\ k_{r d} & 0 & 0 & 0 & -k_{r d} & 0 & -a_{1} & -a_{2} \\ 0 & k_{r t} & 0 & 0 & -k_{r t} & 0 & a_{3} & -a_{4} \\ 0 & 0 & k_{r d} & 0 & -k_{r d} & 0 & a_{1} & -k_{r d} D_{2} \\ 0 & 0 & 0 & k_{r t} & -k_{r t} & 0 & a_{3} & -k_{r t} D_{2} \\ k_{r d} & k_{r t} & k_{r d} & k_{r t} & -a_{5} & k_{p} & a_{6} & a_{7} \\ -a_{12} & a_{13} & -a_{12} & a_{13} & a_{8} & k_{p} D_{1} & a_{6} & a_{9} \\ k_{r d} & k_{r t} & -k_{r d} & -k_{r t} & a_{10} & -a_{10} & 0 & a_{11}\end{array}\right]\left\{\begin{array}{c}x_{r 1} \\ x_{r 2} \\ x_{r 3} \\ x_{r 4} \\ x_{p} \\ x_{0} \\ \theta \\ \beta\end{array}\right\}$

$$
=\left[\begin{array}{cccccccc}
0 & 0 & 0 & 0 & 0 & 0 & 0 & 0 \\
c_{s d} & 0 & 0 & 0 & k_{s d} & 0 & 0 & 0 \\
0 & c_{s d} & 0 & 0 & 0 & k_{s d} & 0 & 0 \\
0 & 0 & c_{s d} & 0 & 0 & 0 & k_{s d} & 0 \\
0 & 0 & 0 & c_{s d} & 0 & 0 & 0 & k_{s d} \\
0 & 0 & 0 & 0 & 0 & 0 & 0 & 0 \\
0 & 0 & 0 & 0 & 0 & 0 & 0 & 0 \\
0 & 0 & 0 & 0 & 0 & 0 & 0 & 0
\end{array}\right]\left\{\begin{array}{c}
\dot{\varphi}(t)_{1} \\
\dot{\varphi}(t)_{2} \\
\dot{\varphi}(t)_{3} \\
\dot{\varphi}(t)_{4} \\
\varphi(t)_{1} \\
\varphi(t)_{2} \\
\varphi(t)_{3} \\
\varphi(t)_{4}
\end{array}\right\}
$$




\section{$\mathbf{X I X}$ \\ CONEMI}

\section{CONCLUSÕES}

A modelagem analítica através da Mecânica Clássica pode representar uma boa aproximação entre a resposta obtida e a real excitação a que o veículo está submetido durante a rodagem. Certamente, há de se considerar as simplificações adotadas pelo modelo, que podem afetar a precisão de resposta - como a consideração de suspensões independentes, algo que não ocorre em todos os veículos; a não consideração da massa dos eixos e das forças de inércia correlatas, assim como a influência destes entre os pares de rodas, representados de maneira totalmente independente no modelo abordado. Há também diversos outros elementos capazes de absorver energia de impacto proveniente da rodagem do automóvel, como os coxins do amortecedor, responsáveis pela afixação da suspensão ao chassi do veículo (ou monobloco), e a própria carroceria, considerada totalmente rígida neste processo de modelagem; a angulação das molas e amortecedores entre a conexão com a bandeja de suspensão (ou braço oscilante) e o monobloco da carroceria, havendo a necessidade de se obter coeficientes de rigidez e amortecimento equivalentes para uma boa representação - fatores estes bem consolidados em bibliografias clássicas da área automobilística. No entanto, para o que aqui se propôs, a abordagem e resultados obtidos são suficientemente satisfatórios como ponto de partida na realização de estudos mais específicos, dado que os componentes principais de massa, rigidez e amortecimento presentes em um veículo foram representados, assim como o nível considerável de graus de liberdade para a representação do modelo, fato observado, principalmente, pela robustez da representação matricial das equações de movimento. Além do mais, através da discriminação entre propriedades de rigidez e amortecimento das partes dianteira e traseira do veículo, realizada pelo presente modelo, é possível se analisar situações como a influência da diferença de calibragem dos pneus na resposta obtida, assim como o estudo de modelos que levem em consideração a diferença de requisição de carga entre as partes, situação recorrente em veículos pesados e que implica na necessária alteração de propriedades entre conjuntos mola/amortecedor dianteiros e traseiros. 


\section{$\mathbf{X I X}$ \\ CIONEMI}

\section{REFERÊNCIAS}

RAO, Singiresu S. Vibrações Mecâncias. 4ạ ed. São Paulo: Pearson Prentice Hall, 2008.

RIPPER NETO, Arthur Palmeira. Vibrações Mecâncias. 1ạ ed. Rio de Janeiro: E-papers Serviços Editoriais, 2007.

IIDA, Itiro; GUIMARÃES, Lia Buarque de Macedo. Ergonomia: Projeto e Produção. 3a ed. São Paulo: Blucher, 2018.

CORRÊA, Vanderlei Moraes; BOLETTI, Rosane Rosner. Ergonomia: Fundamentos e Aplicações. 1a ed. Porto Alegre: Bookman, 2015.

SOLETO Jr, José; FRANÇA, Luís Novaes Ferreira. Introdução às Vibrações Mecânicas. 1a ed. São Paulo: Blucher, 2006. 